\title{
Asthma and pulmonary embolism: bringing airways and vessels closer together
}

\author{
Stavros V. Konstantinides
}

Affiliations: Center for Thrombosis and Hemostasis (CTH), University Medical Center Mainz, Mainz, Germany.

Correspondence: S.V. Konstantinides, Center for Thrombosis and Hemostasis, Johannes Gutenberg University Medical Center, Langenbeckstrasse 1, Bldg. 403, 55131 Mainz, Germany.

E-mail: stavros.konstantinidesQunimedizin-mainz.de

0

@ERSpublications

Better knowledge of inflammation teaches us that airways and the circulation are much closer than we thought http://ow.ly/szr9Q

In the present issue of the European Respiratory Journal, CHUNG et al. [1] report the results of a longitudinal epidemiological study in Taiwan which adds significant evidence for the existence of a link between asthma and the risk of developing pulmonary embolism (PE). The authors are to be congratulated for following 31356 asthmatic patients for a total of 186182 patient-years and matching them with 125157 nonasthmatic individuals (743374 person-years). This is relevant, truly representative epidemiological work, as it appears to include the entire (99\%) population diagnosed with asthma in this country. Its results will hopefully have far reaching implications, helping to finally eliminate the distance between the airways and the (pulmonary) vessels, which is still astronomical in the minds of many pulmonologists and vascular specialists.

Venous thromboembolism (VTE), encompassing both deep vein thrombosis (DVT) and acute PE, is the third most frequent cardiovascular disease with an overall annual incidence of 1-2 per 1000 population $[2,3]$. Its most threatening clinical manifestation, acute PE, is a major cause of mortality, morbidity, and hospitalisation in Europe. The magnitude of the problem is supported by an epidemiological model which estimated that up to 317000 deaths were related to VTE in six countries of the European Union (with a total population of 454.4 million) in 2004 [3]. It is important to emphasise that, in the vast majority of early PE-related deaths, the disease remains undiagnosed during life due either to a fulminant clinical course or, apparently, to a low level of awareness and clinical suspicion.

Until now, most of the existing data on the epidemiology and risk factors of PE have been derived from studies that examined VTE as a whole [4, 5]. Apart from the well-known factors listed in existing guidelines [6], inflammation has increasingly been recognised as a predisposing condition for the development of venous thrombosis and its consequences. Recent findings indicate that respiratory, urinary tract, skin and abdominal infections, as well as sepsis are related to an almost doubling of the risk to develop VTE [7]. Patients with inflammatory bowel disease also have an increased risk of VTE, which is now considered a relatively common extra-intestinal manifestation of ulcerative colitis and Crohn's disease [8] and accounts for increased inpatient mortality as well as higher hospital costs [9]. Furthermore, cohort studies from the UK [10] and Sweden [11] confirmed an association between DVT/PE and a large number of immunemediated disorders. Clearly, systemic inflammation is associated with both a procoagulant state and damage

Received: Jan 132014 | Accepted: Jan 132014

Support statement: The work of S.V. Konstantinides was supported by the Federal Ministry of Education and Research (BMBF 01EO1003). The author is responsible for the contents of this publication.

Conflict of interest: None declared.

Copyright @ERS 2014 
to the vessel wall. Aside from the well described effects of numerous inflammatory cytokines, more recently discovered pathomechanisms include microparticles [12] and neutrophil extracellular traps (NETs). The former are derived from immune (and other) cells; they accumulate at sites of vascular injury and can act as procoagulants, expressing tissue factor, among others. The latter are structures of chromatin (DNA/ histones) and antimicrobial proteins derived from polymorphonuclear cells. NETs are actively expelled into the extracellular space during bacterial infections and inflammation [13]; they activate the intrinsic pathway of coagulation and may initiate the formation of venous or arterial thrombi [14].

Let us return to the lungs, focusing on inflammatory airway diseases. Chronic obstructive pulmonary disease (COPD) is a major health burden worldwide, and may become the fourth or even third leading cause of death within the next two decades $[15,16]$. COPD is a risk factor for PE, with the prevalence of PE being as high as $25 \%$ in patients with exacerbations of COPD [17, 18]. This finding may be of particular importance, as acute undiagnosed PE may contribute to COPD-related mortality. In fact, most COPDrelated deaths are due to exacerbations, 30\% of which are of "unknown" cause [19]. Recently, MAJOoR et al. [20] investigated the relationship between asthma of different severities and VTE in a cross-sectional study with an external reference population. In 684 patients, 283 of whom had severe asthma and 365 of whom had mild-to-moderate asthma, the incidence of PE was 0.93 per 1000 patient-years in patients with severe asthma compared with 0.33 per 1000 patient-years in those with mild-to-moderate asthma and only 0.18 per 1000 patient-years in the reference population. This corresponded to standardised rate ratios of 8.93 and 3.97 for severe and mild-to-moderate asthma, respectively! Asthma severity was, besides oral corticosteroid use, an independent predictor of PE in asthma patients. In contrast to the impact on PE, patients with asthma did not exhibit a significant increase in the incidence of DVT compared with the control group [20]. Although less impressive, the results of the study by CHUNG et al. [1] now confirm, complement and extend the findings of MAJOOR et al. [20], showing a hazard ratio for PE of 3.24 in the asthmatic cohort compared with non-asthmatic controls; the rate of DVT was not examined in the present study.

The message of the study by CHUnG et al. [1] is consistent with that of the study by Majoor et al. [20] and with the studies on patients with COPD: inflammatory airway diseases are a predisposing or risk factor for PE, perhaps more so than for DVT. All these observations suggest that both systemic and local inflammatory mechanisms may link inflammation of the airways and thrombosis, and that perhaps some of the reported cases may be due to in situ pulmonary arterial thrombosis rather than VTE. Whatever the predominant pathomechanism, the implications for clinical practice are quite clear: physicians can no longer afford to "compartmentalise" in their minds the diseases of the airways and those of the pulmonary circulation. Pulmonologists caring for patients with asthma or COPD should not ignore the effects that these diseases, their severity and their treatment may have on the pulmonary vasculature, and should always think of PE as a possible cause of (or contributor to) the exacerbations with which their patients may present. Conversely, cardiologists and vascular physicians treating patients with acute PE should actively seek a history suggestive of asthma or COPD in their patients. These important comorbidities may affect therapeutic decisions in the acute phase, particularly with regard to the detection and reversal (e.g. with fibrinolysis) of acute right ventricular failure, which may be devastating in patients with pre-existing pressure overload and cardiorespiratory compromise. After the acute phase, they may also affect the referral to pulmonologists for appropriate diagnosis and specific treatment, the duration of anticoagulation for secondary prophylaxis after $\mathrm{PE}$, and the need for primary prophylaxis during exacerbations of asthma or COPD in the future. Better knowledge of inflammation brings our disciplines closer together, and teaches us that airways and the circulation are much closer than we have been thinking all this time.

\section{References}

1 Chung W-S, Lin C-L, Ho F-M, et al. Asthma increases pulmonary thromboembolism risk: a nationwide population cohort study. Eur Respir J 2014; 43: 801-807.

2 Heit JA. The epidemiology of venous thromboembolism in the community. Arterioscler Thromb Vasc Biol 2008; 28: 370-372.

3 Cohen AT, Agnelli G, Anderson FA, et al. Venous thromboembolism (VTE) in Europe. The number of VTE events and associated morbidity and mortality. Thromb Haemost 2007; 98: 756-764.

4 Rogers MA, Levine DA, Blumberg N, et al. Triggers of hospitalization for venous thromboembolism. Circulation 2012; 125: 2092-2099.

Anderson FA Jr, Spencer FA. Risk factors for venous thromboembolism. Circulation 2003; 107: I9-I16.

6 Torbicki A, Perrier A, Konstantinides SV, et al. Guidelines on the diagnosis and management of acute pulmonary embolism: the Task Force for the Diagnosis and Management of Acute Pulmonary Embolism of the European Society of Cardiology (ESC). Eur Heart J 2008; 29: 2276-2315.

7 Schmidt M, Horvath-Puho E, Thomsen RW, et al. Acute infections and venous thromboembolism. J Intern Med 2012; 271: 608-618.

8 Murthy SK, Nguyen GC. Venous thromboembolism in inflammatory bowel disease: an epidemiological review. Am J Gastroenterol 2011; 106: 713-718. 
9 Nguyen GC, Sam J. Rising prevalence of venous thromboembolism and its impact on mortality among hospitalized inflammatory bowel disease patients. Am J Gastroenterol 2008; 103: 2272-2280.

10 Ramagopalan SV, Wotton CJ, Handel AE, et al. Risk of venous thromboembolism in people admitted to hospital with selected immune-mediated diseases: record-linkage study. BMC Med 2011; 9: 1.

11 Zöller B, Li X, Sundquist J, et al. Risk of pulmonary embolism in patients with autoimmune disorders: a nationwide follow-up study from Sweden. Lancet 2012; 379: 244-249.

12 Distler JH, Huber LC, Gay S, et al. Microparticles as mediators of cellular cross-talk in inflammatory disease. Autoimmunity 2006; 39: 683-690.

13 Brinkmann V, Reichard U, Goosmann C, et al. Neutrophil extracellular traps kill bacteria. Science 2004; 303: 1532-1535.

14 Fuchs TA, Brill A, Wagner DD. Neutrophil extracellular trap (NET) impact on deep vein thrombosis. Arterioscler Thromb Vasc Biol 2012; 32: 1777-1783.

15 Mathers CD, Loncar D. Projections of global mortality and burden of disease from 2002 to 2030. PLoS Med 2006; 3: e442.

16 Murray CJ, Lopez AD. Mortality by cause for eight regions of the world: Global Burden of Disease Study. Lancet 1997; 349: 1269-1276.

17 Tillie-Leblond I, Marquette CH, Perez T, et al. Pulmonary embolism in patients with unexplained exacerbation of chronic obstructive pulmonary disease: prevalence and risk factors. Ann Intern Med 2006; 144: 390-396.

18 Rizkallah J, Man SF, Sin DD. Prevalence of pulmonary embolism in acute exacerbations of COPD: a systematic review and metaanalysis. Chest 2009; 135: 786-793.

19 Sapey E, Stockley RA. COPD exacerbations. 2: aetiology. Thorax 2006; 61: 250-258.

20 Majoor CJ, Kamphuisen PW, Zwinderman AH, et al. Risk of deep vein thrombosis and pulmonary embolism in asthma. Eur Respir J 2013; 42: 655-661. 